\title{
Tunnel Face Image Segmentation Optimization
}

\author{
http://dx.doi.org/10.3991/ijes.v2i2.2739 \\ DU Cheng ${ }^{1}$, LENG Biao ${ }^{2}$ \\ ${ }^{1}$ Southwest University for Nationalities of China, Chengdu \\ ${ }^{2}$ Southwest Jjiaotong University of China Chengdu
}

\begin{abstract}
With the development of Transportation Highway and railroad build, mining tunnel geological exploration in the road construction in the proportion of great. This paper presents a design of image processing software of Geological Engineering images for automatic analysis and processing. At present, the technology of image processing, most algorithms are based on the specific image information of specific analysis, and the face image is very complicated, different regions, and even the same construction sections in different areas of the face image may have very big difference. For the tunnel excavation face of digital image processing algorithms have little, need to start from scratch. This paper describes the use of digital image processing technology of Geological Engineering image image segmentation, found on the rock face, through the comparison of edge detection operator and Sobel Gauss - Laplasse operator methods advantages and disadvantages, a value of two images as the processing object image processing algorithm. The technology of Geological Engineering image analysis on tunnel construction period prediction plays a very important role.
\end{abstract}

Index Terms-Image division, Geological Engineering, Imagery processing

\section{INTRODUCTION}

As our country increase the infrastructure construction, network construction will become one of the focal points of investment. Because the geographical location of many places, the tunnel occupies a significant proportion in line, and individual can be up to $40-50 \%$ or more. So the mining tunnel construction and geological detection in the railway construction occupies an extremely important role.

The excavation of the tunnel construction will inevitably encounter the Geological Engineering and the amount of excavation (including super underbreak) measurement issues and force structure changes, to predict the occurrence and geological structure of the tunnel in front of the crack and the tunnela series of questions. This need to create a three-dimensional model of the tunnel .It is

almost impossible to achieve by manually drawing. Domestic research in this area is very few. This article is to automatically analyze and process an image processing software for the design of the Geological Engineering image tunnel geological 3D model, and the geological conditions of the tunnel in front of inference. Some key technology of the projects can fill the gaps in the field. Also it has great significance in the realization of tunnel rock formations geological structure detection and prediction in domestic . This paper focuses on the image analysis section in the image processing software analysis. one can be optimized and practical image analysis algorithms.

\section{THE QUALITY EVALUATION OF GEOLOGICAL ENGINEERING IMAGE}

The Geological Engineering digital image from the tunnel construction are different according to the geographical position, construction progress, builder and other anthropogenic factor. The Geological Engineering digital image acquied from the different areav construction plot may different as the different geologic structure, so as the mehod of different kind of iamge processing is different. Thus,before the processing of image, it is necessary to evaluate the quality of image so as to reduce the influence caused by the quality problems of input image . Generally speaking, the Geological Engineering digital image which fit to processing should meet the following conditions:

The image should have some brightness

When shoting, the flashlight need some intensity. If the intensity is not enough, the gray value of digital image will low and it has no processing value.

The image should clear

The clear image will keep the radio-frequency component of the stratified rock boundary, divisional plane、 crack in image. If the image is not clear, when processing the image automaticly ,a lot of marginal information will lost.

Try to avoid mass of image noise

The noise of Geological Engineering image causd by the Camera Products. The noise of image is more, the processing is more difficult. As the joint wall and hair side of tunnel cavity wall have different reflection level for optical line in practical engineering, so the optical noise is different.

\section{THE FUNDAMENTAL FEATURE OF GEOLOGICAL ENGINEERING DIGITAL IMAGE}

The Geological Engineering digital image is different from other image. It has feature itself. In the Geological Engineering image, the boundary of different terrane is clear, and the edge gradient is big. Some boundaries terrane of Geological Engineering image is not clear enough. Slippage of terrane and terrane divisional plane will turn out some boundaries. Veins of target and background are not even. There are lots of cracks which are different in size and area in image. If some big particles have color similar to the background or even brighter, it can lead to the change of crack image color. But overall, cracks are brighter than background color. Some cracks are very short and thick,and interlaced with each other. As the characteristics of digital camera, the brightness is not even in Geological Engineering digital image. The shape of boundaries and cracks is no immovable plus some tun- 
nel builder and oter anthropogenic factor. This turn out lots of fake boundaries and made the complicated Geological Engineering digital image more complicated and hard to process. So it can not use one special algorithm to process the Geological Engineering image, but need make a concrete analysis of concrete problems .

\section{AIRSPACE TRANSFORMATION TO STRENGTHEN}

\section{(1) Histogram equalization}

In grey level value statistic column diagram, image is one discrete function of 1-D:

$$
\begin{aligned}
& p\left(s_{k}\right)=n_{k} / n \\
& k=0, \quad 1, \quad \ldots, L-1
\end{aligned}
$$

In (1-1), ${ }^{S_{k}}$ is the k Gray Level of image $\mathrm{f}(\mathrm{x}, \mathrm{y}), n_{k}$ is the number of pels which is with Gray Level $s_{k}$ in $\mathrm{f}(\mathrm{x}, \mathrm{y}) . \mathrm{n}$ is the sum of image pels. $p\left(s_{k}\right)$ is the estimate to the probability of occoriginal drawingurrence of

$S_{k}$.So the column diagram to provide the distribution situation of original drawing gray level ,that is put forward a whole description of all the gray level in drawing .Accodring to the analysis of corresponding column diagram which come from different image from the same scene, it found if the image is brighter or darker, the dynamic range of column diagram is small. And the dynamic range of image is larger, the image effect is better . It can change the shape of column diagram to stength the effect of picture contrast. This method is basd on probability theory. The common methods are column diagram equalization and column diagram specification .

The basic thought of histogram equalization is to change the column diagram of original drawing into the form of homogeneous distribution. In this way, it can large the dynamic range of pixel gray value and thus achieve the effect of stength the image overall contrast . Now the histogram character function of grey level value statistic list in the following :

$$
\begin{gathered}
p_{s}\left(s_{k}\right)=n_{k} / n \\
0 \leq s \leq L-1, \quad k=0, \quad 1, \quad \ldots, \quad L-1
\end{gathered}
$$

$p_{s}\left(s_{k}\right)$ represent the probability of occurrence of $\mathrm{k}$ gray level in original drawing.

$s_{k}$ is independent variable. The curved shape which as $p_{s}\left(s_{k}\right)$ for function is the column diagram of image.

The histogram equalization in fact is to process the point in image. The strengthen function need to meet the following two condition :

1) $\mathrm{EH}(\mathrm{s})$ 在 $0 \leq \mathrm{s} \leq \mathrm{L}-1$ one monodrome function ;

$$
\text { 2) 对 } 0 \leq s \leq L-1 \text { 有 } 0 \leq E H(s) \leq L-1 \text { 。 }
$$

The first condition makes sure each grayscale in original drawing keeping the sequence from back to white (or from white to back ) after changing. The second condition makes sure the dynamic range of gray value consistency before and after the transformation .
Cumulative distribution function( CDF ) meet the two conditions and can change the $\mathrm{S}$ distributed to transform to $t$ homogeneous distribution. In fact,

CDF of $\mathrm{s}$ is the cumulative histogram of original drawing. Now,

$$
\begin{gathered}
t_{k}=E H\left(s_{k}\right)=\sum_{i=0}^{k} \frac{n_{i}}{n}=\sum_{i=0}^{k} p_{s}\left(s_{i}\right) \quad 0 \leq s \leq 1, \\
k=0, \quad 1, \quad \ldots, \quad L-1 \quad \text { (1-3) }
\end{gathered}
$$

By the above formula, according to the original column diagram can work out the gray value of each pixel after equalization directly. In practice, tk should trunc to meet the requirement of digital image.

\section{THE PROCESSING OF GEOLOGICAL ENGINEERING IMAGE}

Image segmentation is the process that according to certain rules image is divided into several subsets .It is a critical step in image processing. The purpose is to extract the significant characteristics or interested object from image .It will be possible to make the image feature extraction, parameter measurement and higher analysis and understanding. Edge detection is in the image segmentation based on boundary treatment. The first step is to deal with the image edge detection. In the image processing system, used in edge detection algorithm mainly includes the following several kinds: sobel operator and gauss - Laplace operator. Diagram $1 \mathrm{c}$ ) is the figure 1 a) application sobel convolution template operation results. The sobel operator are two 3 by 3 convolution kernels, through the two small area template in the image of the mobile, complete image of each pixel point with the two convolution nuclear convolution operation, and finally the edge of the output amplitude results can detect the edge of the image. Below is the figure 1 a) with gaussian - Laplace convolution template processing results.



a) original image



b) Sobel convolution moulding board 


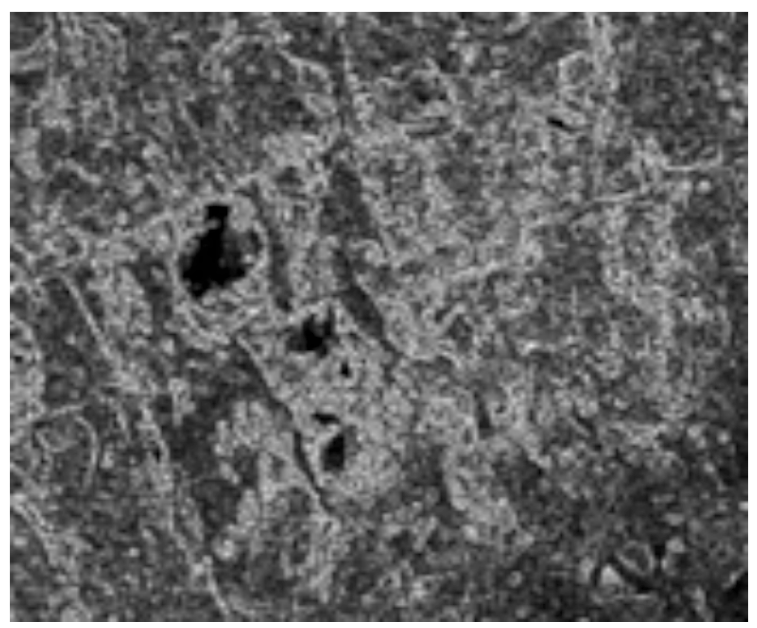

c) the processing result

Figure 1. the results of sobel arithmetic operators process the iamge.

\begin{tabular}{|c|c|c|c|c|}
\hline-2 & -4 & -4 & -4 & -2 \\
\hline-4 & 0 & 8 & 0 & -4 \\
\hline-4 & 8 & 24 & 8 & -4 \\
\hline-4 & 0 & 8 & 0 & -4 \\
\hline-2 & -4 & -4 & -4 & -2 \\
\hline
\end{tabular}

a) gaussian - Laplace convolution moulding board

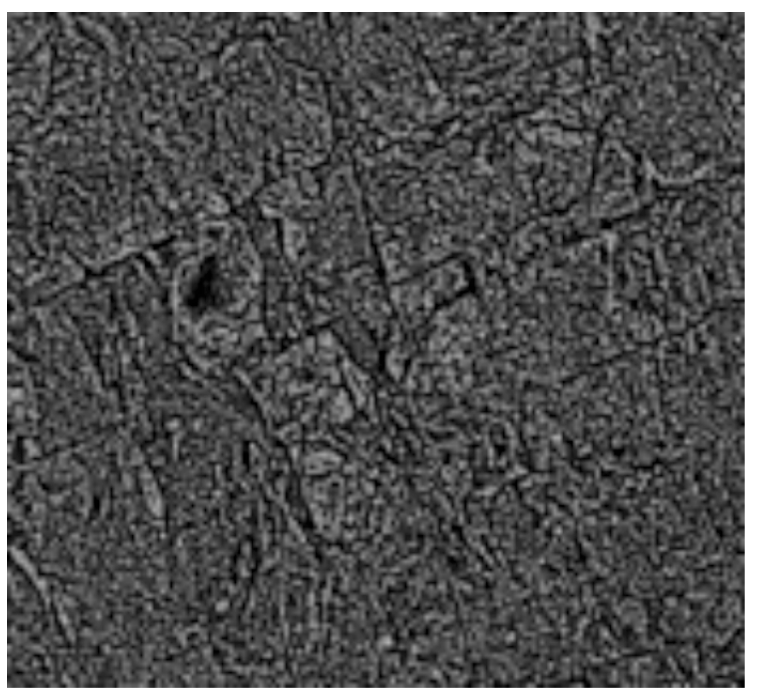

b) the processing result

Figure 2. the results of gaussian - Laplace convolution moulding board

From the processing results, we can see among the original image application different convolution template, the sobel convolution template processing results, the processed image is the worst .The interference in the boundary of the image is still very large. But gaussian - Laplace convolution form the processed images the border has basically been able seen significant.

\section{THE ALGORITHM OF FACE IMAGE PROCESSING}

The technology of image processing has its special feature. Now, the image processing technology, most algorithms are based on the digital image information specific analysis. These algorithms are often only applies to certain scope, and not in any case can be used. It also has a certain difficulty to process the tunnel working face digital image. Especially working face image is very complicated. And different regions, even the same construction lots of different parts of the working face digital image may have very big difference. According to the tunnel working face of digital image processing algorithm almost no for concrete tunnel working face digital image of the kinds of analysis and adopt what kind of algorithm is a unknown, we need to start from scratch, thus the difficulty of processing is obvious.

Therefore we propose in image processing system, to binary image for processing object, through a series of set operation, improve the image processing effect. Binary morphology of the operand is set, the basic morphological operation is corrosion and expansion. Corrosion is to eliminate the object image yuan with point of a kind of process, the result that the rest of the objects along the perimeter before than processing small several pixel area. If the object is round, its diameter in every time after corrosion will reduce a few pixels. From a picture of corrosion on the segmentation images small and medium-sized and meaningless image element set it is useful. Inflation is simple with the object of the contact point of all the merger of the object of the process. The results of the process is to make the object area increases the corresponding number of pixels. If the object is round, its diameter increased a few pixels in every time after inflation. If two objects are in a little less than the above several pixels, then they will through the expansion connected up. Inflation in the fill a void in the object after segmentation is very useful. A general sense of the corrosion defined as

$$
E=B \Theta S=\left\{x, y \mid S_{x y} \subseteq B\right\}
$$

Namely the $\mathrm{S}$ to $\mathrm{B}$ corrosion generated binary image $\mathrm{E}$ is this point $(\mathrm{x}, \mathrm{y})$ set, if $\mathrm{S}$ origin displacement to $(\mathrm{x}, \mathrm{y}), \mathrm{S}$ will completely in B in.

The general meaning of the definition for inflation

$$
D=B \oplus S=\left\{x, y \mid S_{x y} \cap B \neq \phi\right\}
$$

That is to say, $\mathrm{S}$ to $\mathrm{B}$ expansion generated binary images is $\mathrm{D}$ by this point $(\mathrm{x}, \mathrm{y})$ form, if $\mathrm{S}$ origin shift to $(\mathrm{x}, \mathrm{y})$, then it and $\mathrm{B}$ intersection not empty.

After the first corrosion expansion process called open operation. It can eliminate small objects, in fine point separation objects and smooth larger boundary and not obviously change the area. The definition of the open operation for

$$
B \circ S=(B \Theta S) \oplus S
$$

After the first expansion corrosion process called closed operation. It can pack object interior tiny hole, and connect adjacent objects, also smooth the boundary under the condition of not obvious change area, defined as

$$
B \cdot S=(B \oplus S) \Theta S
$$

Usually, when there is noise in the image threshold binarization, the binary image of the target area boundary is often not smooth. The target area has some mistake be- 
cause formed hole, background area because there are some mistake and formation of granular noise. Continuous start-up and closed operation can significantly send this kind of situation. Sometimes after a few times continuous corrosion iteration, and several times the same number of inflation, produce the desired effect. Figure 3 is a expansion, corrosion example.

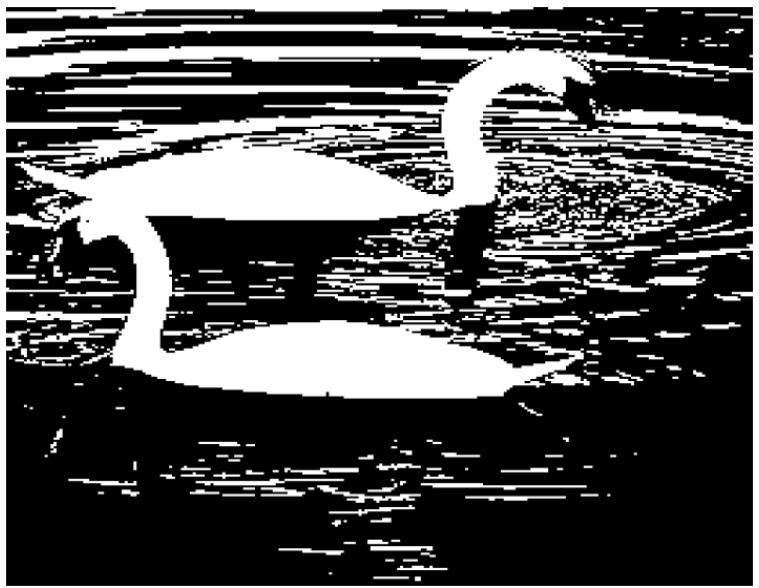

a) the original binary image



b) The results after one open computing

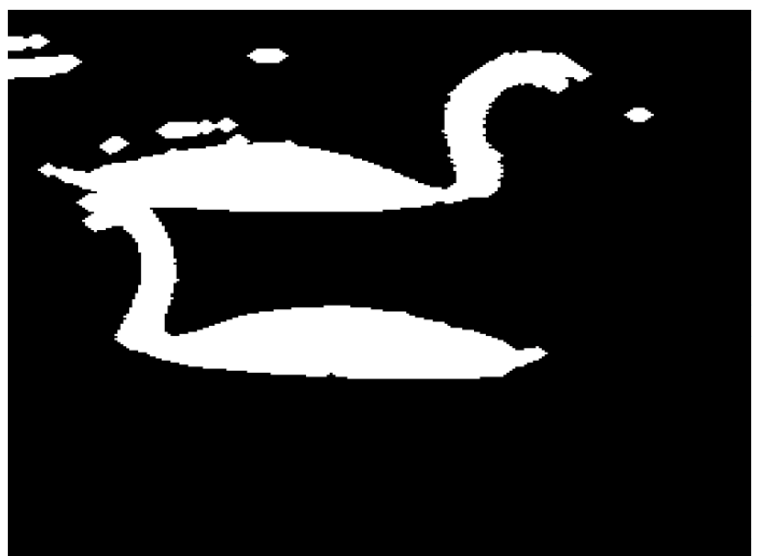

c) The results after three corrosions and expansions

Figure 3. The original image and Morphology processing results for the above results are obviously。

\section{CONCLUSION AND PROSPECT}

This system compare the advantages and disadvantages of different image processing methods based on the analysis of the working face image. It optimizes the existing image and realize an easier operation image processing in computer, and apply to the specific cases. The system provides a concrete realization method for the application for the modern computer image processing technology to advance geological forecast, the comprehensive prediction made material to carry on the analysis and research, timely and accurate grasp of the tunnel in front of geology and improve the construction of geological hazard in the strain capacity of the image processing system. It will make use of modern technology to play in the tunnel construction of the tunnel, and to accomplish the leap.

\section{REFERENCES}

[1] Soe-Sen Leu, Shiu-Lin Chang. Digital image processing based approach for tunnel excavation faces [J]. Automation In Construction, 2005, 14:750-765 http://dx.doi.org/10.1016/j.autcon.2005.02. $\underline{004}$

[2] Liu Yang, Tunnel working face image processing system research and implementation [D]; Southwest jiaotong university, 2007,

[3] DuCheng; LengBiao, MaBo geological image feature extraction [A]; 2009 international information technology and application memoir [C]; 2009,

[4] Cuiyin Liu, Xiuqiong Zhang, Xiaofeng Li, Yani Liu, Jun Yang ,Gaussian Kernelized Fuzzy c-means with Spatial Information Algorithm for Image Segmentation, ournal of Computers, Vol 7, No 6 (2012), 1511-1518, Jun 2012

[5] Zhen Hua, Yewei Li, Jinjiang Li,Image Optimization Algorithm Based on Salient Region and Layout Adjustment, Journal of Computers, Vol 6, No 6 (2011), 1222-1229, Jun 2011

[6] Rong Zhu, Yong Wang, Application of Improved Median Filter on Image Processing, Journal of Computers, Vol 7, No 4 (2012), 838-841, Apr 2012

[7] Xuebo Jin, Jia Bao, Jingjing Du,Image Enhancement Based on Selective - Retinex Fusion Algorithm, Journal of Software, Vol 7 , No 6 (2012), 1187-1194, Jun 2012

[8] Jun Zhi, Jian-yong Liu, Hui Yuan, Guang-hua Wu,Design of Simulated Pattern Painting Based on Image Segmentation and Recognition Method, Journal of Software, Vol 6, No 11 (2011), 2232-2238, Nov 2011

[9] Bing Zhu, Xuyan Zhou, Bin Tan, Xue-mou Liu,Algorithm for Detecting the Image of River Sediment Based on Hydrometric Cablewa, Journal of Software, Vol 6, No 8 (2011), 1437-1444, Aug 2011

\section{AUTHORS}

DU Cheng is with College of Electric \& Information Engineering, Southwest University for Nationalities of China, Chengdu

LENG Biao is with School of Civil Engineering, Southwest Jjiaotong University of China Chengdu

Submitted 06 May 2013. Published as re-submitted by the authors 12 May 2014. 